\title{
Towards a Taxonomy of API Services in Logistics
}

\author{
Frederik Möller \\ TU Dortmund/Fraunhofer ISST \\ Frederik.Moeller@tu-dortmund.de
}

\author{
Maleen Stachon \\ TU Dortmund University \\ Maleen.Stachon@tu- \\ dortmund.de
}

\author{
Ilka Jussen \\ TU Dortmund University \\ Ilka.Jussen@tu-dortmund.de
}

\author{
Julia Schweihoff \\ TU Dortmund University \\ Julia.Schweihoff@ \\ $\underline{\text { tu-dortmund.de }}$
}

\author{
Hendrik van der Valk \\ TU Dortmund University \\ Hendrik.van-der-valk@ \\ tu-dortmund.de
}

\author{
Michael Schmidt \\ Fraunhofer IML \\ Michael.b.schmidt@ \\ iml.fraunhofer.de
}

\author{
Stephanie Handrup \\ TU Dortmund University \\ Stephanie.Handrup@ \\ tu-dortmund.de
}

\begin{abstract}
Data are a valuable asset for companies in the logistics sector to optimize internally and develop new business models. They can be like a magnifying glass, making previously opaque logistical processes transparent and finding previously hidden optimization potentials. Typical applications are tracking the transport status, route optimization, monitoring pharmaceutical products, or monitoring shocks for fragile cargo along the trade lanes. One way to use data is to tap into publicly or commercially available Application Programming Interfaces (APIs). As a result, logistics service providers can get or provide data automatically via a machine-tomachine interface. However, the landscape of API service providers is vast, unstructured, and intransparent in terms of potential data that companies can leverage. Given their high potential for logistics, the paper proposes a taxonomy of API services in logistics based on the inductive analysis of three API databases.
\end{abstract}

\section{Introduction}

The rapid development of digital technologies leads to increased competition in the logistics service industry, which, as it is, is characterized by low margins and complex competition (as shown by studies of Roland Berger [1] and PwC [2]). To maintain competitiveness and growth, logistics service companies need to digitize and define their strategies and business models based on data and digital technologies [1, 3-5]. Especially since there is a variety of start-ups emerging in logistics that leverage data and propose digital business models to generate visibility, optimize processes, or connect different parties of the logistical supply chain $[6,7]$. Application Programming Interfaces (APIs) offer companies a suitable opportunity to transform their business models and gain a competitive advantage through using data $[8,9]$. They can be accessed to collect data from various sources, combine them, and generate new data-based products and services [10]. Through APIs, companies can enable their customers to access data and services while generating a new source of revenue [11, 12]. For example, Salesforce generates over $50 \%$ of its revenue through APIs, eBay $60 \%$, and Expedia as much as $90 \%$ [9, 13]. Accordingly, the growing number of APIs available on the market is not surprising and enables companies to tap into new fields and ecosystems [13]. Today, the world's largest API database lists over 24,000 APIs (see ProgrammableWeb [14]). A study conducted by $\mathrm{PwC}$ shows that there is no other industry in which data are more relevant than in the logistics sector [15]. Interestingly, the logistics trend radar by $D H L$ sees APIs as an emerging technological key trend that will be of high relevance within the next five years [16]. An illustrative example for APIs as a root for creative business applications is the Google Maps API. It was not designed for specific products but enabled various developers in different fields to integrate and build on its capabilities [10]. Given the importance of APIs for business transformation, companies must “(...$)$ quickly identify APIs that satisfy their functional and non-functional requirements (...)" [17 p. 36].

The managerial motivations mentioned above are complemented by current research. Möller et al. [18] point out the importance of data-driven services in the logistics domain to optimize and foster the visibility of logistical processes. Subsequently, some companies are offering, e.g., route optimization services through APIs [18], which enables logistics companies to optimize addresses in routes through a machine-tomachine interface rather than manually through a graphical user interface [19]. While the study [18] does analyze data-driven business models in logistics, 
the focus is on business models in general - not on APIs.

Generally, taxonomies analyze objects on a highly specific or very abstract level (specific taxonomies are conceptually narrower and encompass a more delimited framework) [20, 21]. In this respect, the taxonomies here are a more detailed explication tailored to APIs. Given the unique properties of APIs, a deeper analysis of this phenomenon is needed, as it has too far-reaching implications for a merely superficial consideration. Resulting, we use taxonomies to give " $(\ldots)$ greater insights in these particular sectors" [22 p. 6]. Yoo et al. [23], who attribute an increasing role to APIs, identified a lack of research about appropriate methodological and technological principles for API design. To address the lack of research, this paper systemizes logistics API services and the data that they offer. Consequently, we see the need to analyze the availability of API-based services in the logistics domain. Our work complements existing classification considering API services (see [7, 18]) and details them with a distinctive in-depth analysis of APIs. We see significant benefits in disclosing the structure of API services on the market and conceptualize them in a classification scheme. Because of the above, our research question is: How to classify API services and related data in logistics industries?

Given that our goal is classification, we pursue the design of a taxonomy since it enables us to "(...) structure or organize the body of knowledge that constitutes a field (...)" [20 p. 65]. A taxonomy is explicitly suitable for that task, as it is based on the inductive classification of empirical objects [24, 25]. In our case, the empirical objects are APIs in logistics, which we draw from three publicly available databases (i.e., ProgrammableWeb, Datarade, and RapidAPI).

The work adds to prior research on the greater field of digital and data-driven business models in the logistics industry $[6,18]$. It enriches them through an in-depth conceptual look at API services $[6,18]$. We follow standard practice in IS research to answer that particular research question and use the method for taxonomy design of Nickerson et al. [26].

The paper is structured as follows: Section 2 defines the key concepts of this research. Section 3 introduces the research approach. The developed taxonomy is presented in the fourth section. We will provide a deeper look at the finer granular taxonomies for the applications track \& trace and route optimization in Section 5. Section 6 concludes the paper with a discussion and an overview of contributions, limitations, and future research.

\section{Background}

\subsection{Data-Driven Services in Logistics}

To conceptualize the notion of data-driven services, we can draw from the field of data-driven business models. Business models, per se, are the explication and reduction of complexity on how a business works [27]. Correspondingly, a data-driven business model is a business model that explicitly leverages data as the central resource to generate value [28-30]. Likewise, in the logistics services industry, these types of business models explicitly leverage data generated through or around the logistical process, i.e., the transport of goods from the point of origin to a destination [18]. Consequently, a data-driven service is a service that focuses on " $(. .$.$) the generation,$ storage, and analysis of data with the ultimate goal to support sensible and better decisions (...)" [31 p. 3]. These data-driven services usually foster visibility into logistical processes or optimize them based on data [18]. A typical example is services for route optimization that collect data from customers about destinations-to-be-visited and return optimized routes based on cost, time, or other parameters [19]. Other examples are providing additional data complementarily to logistics services to enhance decision-making in transport management [32]. Using data from different sources (e.g., Transport Management Systems (TMS)), can have a wide range of positive effects on how business in logistics works [33]. Thus, data are one differentiating parameter distinguishing traditional logistics service providers (i.e., freight forwarders) from digital logistics services providers (i.e., digital freight forwarders) [34].

\subsection{Application Programming Interfaces}

As standardized interfaces between at least two software applications, APIs enable interoperability $[17,35]$. APIs are boundary resources that allow their user to access the capabilities, data, or infrastructure of a third party [36, 37]. The user can thus easily and cheaply reuse existing solutions and benefit from resource savings concerning their development [17, 38]. In addition, the user does not need to know how the API works. The API provider offers all relevant information for API usage, like mechanisms to API access or documentation [39]. A distinction is made between private APIs and public APIs, which are freely accessible (or with minor agreements) [39]. Logistics companies that offer an API often generate revenue for the API through a subscription model or charge a fee per call [18]. 
Wulf \& Blohm provide a review of APIs [40]. They derive archetypes of APIs and divide them into three clusters, the integrators, the free data providers, and the mediators. The first ones provide the user with the opportunity to integrate the APIs in existing system environments, which comes in handy while dealing with existing systems. The free data providers aim at including as many participants as possible without any costs attached, and lastly, the mediators are trying to focus the whole environment around the APIs [40]. The first and third solutions possess industrial relevance for the context of this paper. Nevertheless, APIs are crucial for mediating platforms. However, the relevance of the individual API is often blurry, which requires a decision on each API and their financial relevance [41].

\section{Research Design}

Following the standard procedure in Information Systems (IS) research, we use the method of Nickerson et al. [26] to build the taxonomy. First, the method requires us to formulate a meta-characteristic, i.e., the general purpose of the taxonomy, which is the following: Classification of API services in logistics industries.

Second, as the method is iterative, it calls for choosing ending conditions (subjective and objective), which we also adopt from Nickerson et al. [26]. Third, one must choose between a conceptual-to-empirical (deductive) or empirical-to-conceptual (inductive) approach. Since we collect our data from empirical examples and our taxonomy aims to classify existing API services, we opt for an inductive approach following the empirical-to-conceptual path. We collect our data in three iterations using three different databases and analyze them for the characteristic properties of API services. We included more than one database to compile a potentially diverse and comprehensive sample (see Table 1).

We analyzed the information on each API service in the group of authors. To collect APIs that explicitly provide data in logistics, we use the search term 'logistics' in each database respectively. We manually screened each API service to discuss the suitability of individual objects if necessary. For example, it was not unambiguously clear whether some API services refer to a logistical service, i.e., transporting goods from point A to B. Finally, we distributed the databases over three iterations to incorporate database-specific findings. All three databases vary in the degree of available information on each API. Subsequently, the final taxonomy reflects a common denominator. That means we divided data collection between the authors and discussed the findings per iteration. In the $1^{\text {st }}$ iteration, we analyzed 42 APIs from RapidAPI, followed by the $2^{\text {nd }}$ iteration with 47 APIs from ProgrammableWeb and the $3^{\text {rd }}$ iteration including 37 APIs from DataRade. We selected these databases based on experience and internet search. During each iteration, we held weekly meetings crystallizing relevant API services and corresponding data. Using multiple databases enabled us to mitigate potential bias existing in one demarcated database. We strived to find all relevant APIs and data sources referring to API services and data in logistics during each iteration.

Table 1. Taxonomy design iterations.

\begin{tabular}{|c|l|c|}
\hline Iteration & DataBase & Sample (N) \\
\hline 1 & RapidAPI [42] & 42 \\
\hline 2 & $\begin{array}{l}\text { ProgrammableWeb } \\
{[14]}\end{array}$ & 47 \\
\hline 3 & DataRade [43] $^{1}$ & 37 \\
\hline 4 & Additional Data $^{1}$ & $\ldots$ \\
\hline
\end{tabular}

To design the final taxonomy, we considered two design alternatives. First, various options exist to visualize a taxonomy, e.g., a morphological field, a mathematical set, or a hierarchy [44]. In our case, we visualize the taxonomy hierarchically, as we have identified it as the most comprehensive option for our purpose, to structure and organize [20]. Second, given the complexity in the final hierarchy, we decided to decouple the taxonomy thematically and generate additional taxonomies. Notably, we developed one taxonomy that is on the 'service-level' while complimentary taxonomies are on the 'data-level'. Currently, our taxonomy fulfills the ending conditions partially as prescribed, yet, we see it as a work-inprogress, as it only reflects a snapshot of three databases and, subsequently, only considers an excerpt of available APIs (see Figure 1). The paper reports on the general service-level taxonomy and two 'datalevel'-taxonomies (i.e., for the services route optimization and track \& trace, see Figure 2 and Figure 3).

\section{Taxonomy of API services}

We illustrate the final taxonomy in two parts. First, the 'service-level' taxonomy provides a hierarchical structure of dimensions and characteristics of API services in logistics. Second, we

\footnotetext{
${ }^{1}$ The taxonomy builds on 3 iterations, which we plan to continue in future work.
} 
developed separate hierarchies for the data of two API services, which improved conciseness and drastically reduced the complexity of the taxonomy.

\subsection{Service-Level Taxonomy}

Figure 1 shows the final 'service-level' taxonomy. It consists of three high-level dimensions that distinguish the essential API services at their most abstract level. There is alignment with prior work, highlighting that optimization and visibility are typical data-driven services [18], which we also found in the present study. These dimensions are extended through a $3^{\text {rd }}$ dimension describing general data services. The three dimensions are as follows:

- Visibility Services: Subsumes API services that enable their user to generate visibility into logistical processes. Examples are extracting data about vehicle movement (vehicle tracking), cargo tracking, or collecting the shipment status.

- Planning/Optimization Services: Subsumes API services that refer to activities of planning and optimization. Typical examples are transport optimization or order management.

- Data Services: Subsumes API services that give the user access to data (data access \& consolidation), validation of data (verification \& validation), or capabilities in the form of algorithms (AI algorithm access). Examples are verification and validation of product data or transport data and access to customized maps.

\subsection{Visibility Services}

From our analysis, we found a wide array of APIbased visibility services that we have differentiated threefold (see Figure 1). The first dimension is track \& trace. In that category, we found services that enable different types of tracking of moving goods. These include tracking of vehicles, cargo, and shipment status (see Table 2). APIs enable collecting the status of moving goods, e.g., tracking packages from a courier network or collecting proof of deliveries.

In more detail, specific instances such as sea freight refer to the possibility of locating ocean-bound

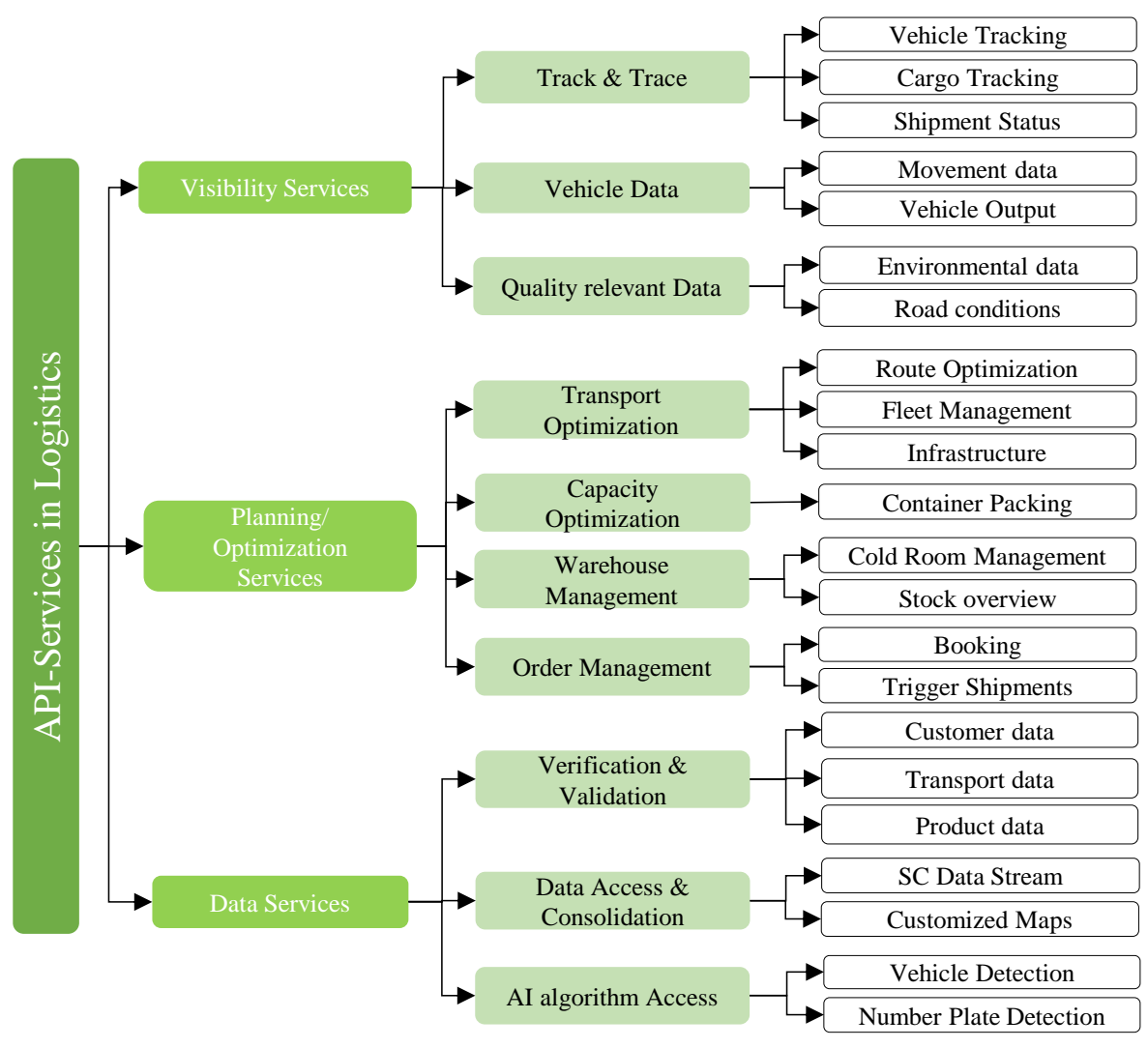

Figure 1. Taxonomy of API services in logistics. 
vessels based on longitudes and latitudes ${ }^{2}$. The API user can extract information such as estimated time of arrivals (ETAs) or departures from these data.

\section{Table 2. Illustrative structure of 'Track \& Trace' services (see Figure 1).}

\begin{tabular}{|c|l|l|}
\hline Service & Sub-Service & Specific Instances \\
\hline \multirow{3}{*}{$\begin{array}{c}\text { Track } \\
\&\end{array}$} & Vehicle & Air Freight, Sea \\
Trace & Tracking & Freight, Ocean Freight \\
\cline { 2 - 3 } & Cargo & Container, Packages \\
& Tracking & \\
\cline { 2 - 3 } & $\begin{array}{l}\text { Shipment } \\
\text { Status }\end{array}$ & $\begin{array}{l}\text { Proof of Delivery, } \\
\text { Automatic Updates, } \\
\text { Tracking Numbers }\end{array}$ \\
\hline
\end{tabular}

Next, APIs enable access to data about vehicles. For example, these include data about the speed or position of a vehicle. Some API service providers offer descriptive data about a vehicle, such as vessels, ports, or historical data ${ }^{3}$. Third, some API services provide information about environmental parameters during the transport process used to ensure the quality of the cargo or goods. Typical services are monitoring of temperatures, air pressure, or humidity (see Table 3).

\section{Table 3. Illustrative structure of 'Quality Relevant' services (see Figure 1).}

\begin{tabular}{|l|l|l|}
\hline Service & Sub-Service & Specific Instances \\
\hline $\begin{array}{l}\text { Quality } \\
\text { Relevant }\end{array}$ & Environmental & Air Pressure, Humidity, \\
& Data & $\begin{array}{l}\text { Temperature, Air } \\
\text { Pollutant Emission }\end{array}$ \\
\cline { 2 - 3 } & Road Data & Shock, Vibration \\
\hline
\end{tabular}

\subsection{Planning/Optimization Services}

The dimension refers to services used to plan or optimize logistical processes. Én detail, the dimension includes route optimization, capacity optimization, warehouse management, and order management.

APIs can tap into digital capabilities for route optimization and tour planning in transport optimization. Typically, the advantage of using APIs to optimize routes contrary to web-based Graphical User Interfaces (GUI) [12] is the volume and speed they can be analyzed and returned. For example, the APIs of Geoapify ${ }^{4}$ or TrackRoad 5 enable the user to calculate thousands of routes in a short period. From those data, users can generate dynamic maps with optimized routes.

\footnotetext{
${ }^{2}$ See https://datalastic.com/developers-documentation/realtimeship-tracking-api/ last-accessed: 11-06-2021

${ }^{3}$ See https://datalastic.com/platform/ last-accessed: 11-06-2021

${ }^{4}$ https://www.geoapify.com/maps-api last-accessed: 29-08-2021
}

Other optimization services include the optimization and matching of freight availability and truck capacity. We can differentiate a distinct segment that enables the management of logistical processes.

\subsection{Data Services}

The category contains all services that we could not categorize directly under track \& trace or route planning/optimization, but that offer data-driven logistics services (e.g., verification of data). For example, the dimension refers to heightening data quality, as these APIs enable users to validate data for correctness. Some APIs offer services to generate and validate barcodes or verify addresses. Since logistical services rely on correct start and end destinations, automatic verification of address data is essential. These services are not clearly categorized as optimization and visibility, so we classify them under data services extending the service categorization of [18].

\section{Table 4. Illustrative structure of 'Verification \& Validation' services (see Figure 1).}

\begin{tabular}{|c|l|l|}
\hline Service & Sub-Service & Specific Instances \\
\hline \multirow{4}{*}{$\begin{array}{c}\text { Verifi- } \\
\text { cation \& } \\
\text { Validation }\end{array}$} & $\begin{array}{l}\text { Customer } \\
\text { Data }\end{array}$ & $\begin{array}{l}\text { Names, Contacts, } \\
\text { Addresses, E-Mail, } \\
\text { Phone Numbers }\end{array}$ \\
\cline { 2 - 3 } & Product Data & $\begin{array}{l}\text { Product Titles, } \\
\text { Prices, Reviews, } \\
\text { Manufacturer }\end{array}$ \\
\cline { 2 - 3 } & Transport & $\begin{array}{l}\text { Estimated Shipping } \\
\text { Costs, Shipping } \\
\text { Information, } \\
\text { CarrierID, SellerID, } \\
\text { OrderID, ParcelID, } \\
\text { ContainerID }\end{array}$ \\
\hline
\end{tabular}

Significantly, the first characteristic refers to the verification \& validation of data in logistics, such as customer data, transport data, or product data (see Table 4). Additionally, the dimension subsumes services to acquire customized maps through APIs or get access to data streams from the supply chain, such as logistical standards. Lastly, the dimension includes services to tap into the capabilities of algorithms. In our case, these refer to AI algorithms used to identify

\footnotetext{
${ }^{5}$ See https://doc.trackroad.com/APIFeatures.aspx\#0 last-accessed: 11-06-2021
} 


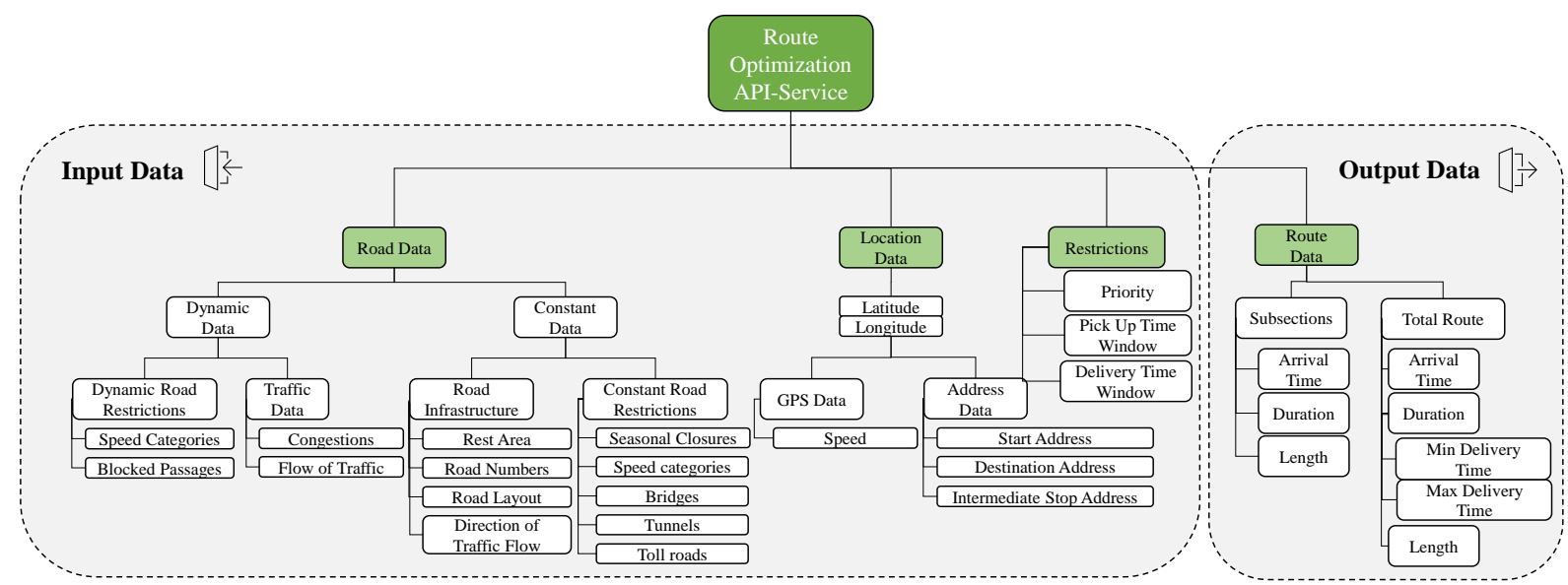

Figure 2. Taxonomy of data for route optimization API services.

different types of objects, such as vehicles or number plates.

\section{Data Taxonomies}

We complement the 'service-level' taxonomy (see Figure 1) through two additional finer-detailed taxonomies. The purpose being that only by decoupling the taxonomies and defining a link through services and data can we foster understandability. We focused on two lower-threshold taxonomies because of the many services and types of data, i.e., for route optimization and track \& trace. These services are the most established and widespread in logistics [18]. We illustrate specific instances of API services and their application with selected illustrative examples. Developing more detailed taxonomies for other services (e.g., capacity optimization) is a task yet to do.

\subsection{Route Optimization}

In the following, we have a closer view of the exemplary data relevant for route optimization processes. Figure 2 shows data for route optimization API services as found in our sample. We divide the data dichotomously into two categories. First, input data that logistics service providers can use to enhance services for route optimization (e.g., data about road infrastructure). Second, into output data such as minimal or maximal delivery time. The data are also divided fourfold into the categories road data, location data, restrictions, and route data (see Figure 2). Road data includes two sub-categories, i.e., dynamic and constant data. Dynamic data are dynamic road restrictions, such as speed limits or blocked passage points. Traffic data include congestions and traffic flow data. Given their dynamic nature, these data are only valid for a dedicated period and can be used before optimizing routes to enhance the quality. Some data are available a priori. For instance, we can assume that road works are announced far in advance, while other data are available on short notice, such as traffic jams. Complementarily, some APIs offer consistent road data. These are, for example, infrastructure data and data describing permanent restrictions. In terms of infrastructure, these data include information about rest areas, road designations, and layouts, as well as permanent directions of allowed traffic flows. Additionally, the data reflect continuously valid restrictions, such as seasonal closures, speed limits, or the weight and height limitations of tunnels and bridges. Thus, these data types can be seen as a kind of master data for the road network and potentially are the foundation for route optimization.

The $2^{\text {nd }}$ category - location data - comprise different types of data, such as latitudes and longitudes. In addition, they originate from two data sources, which diverge in those that move and those that are stationary. For instance, GPS data often includes additional information exceeding geographical localization, such as the speed of the moving vehicle. Contrarily, stationary data contain points of origin, intermediate stops, and destination addresses.

The $3^{\text {rd }}$ category - restrictions - does not focus on route restrictions but surrounding environmental restrictions. We identify three types of restrictions, i.e., priority, pickup time, and delivery time window (see Figure 2). These three types of restrictions originate from the logistical assignment and the partners in the operative process. The time windows of pickup and delivery are directly dependent on the vendor and customer. Priority has to consider three different views, i.e., internal priority lists of the 
transport good, the preferences of the vendor, and the destination partner.

Lastly, the $4^{\text {th }}$ category is route data. The category splits routes into subsections in more detail and subsumes data on arrival times, durations, and sector lengths for each subsection. These subsections add up to the complete route. Ranges within the subsections may add up to minimal and maximal duration, and hence, delivery times.

The task of optimizing the individual route is seldomly part of an API. Instead, in the portrait cases, the API provides the gateway between the physical system, i.e., the data input devices, the application, which fulfills the optimization task, and again, the physical system as an output interface.

\subsection{Track and Trace}

In this section, we detail our analysis regarding data for the track \& trace API services. Track \& trace connects to a plethora of different data categories. Therefore, we sort them loosely in meta-dimensions, based on participant and transportation categories (see Table 5).

\section{Table 5. Categories in Track \& Trace API services.}

\begin{tabular}{|l|l|}
\hline Categories & Data \\
\hline Transport & $\begin{array}{l}\text { Transportation Data, Time Data, Event } \\
\text { Data, Tracking Number }\end{array}$ \\
\hline Participant & $\begin{array}{l}\text { Origin Data, Notifications Data, } \\
\text { Destination Data }\end{array}$ \\
\hline
\end{tabular}

The category transport includes those data relevant during the physical transportation process in tracking and tracing a product. On the other hand, the category participant contains those data that refer to participants of the physical transportation process, e.g., contact information about the origin of the transport or the destination.

\section{Table 6. Illustrative structure of 'Track \& Trace' data (see Figure 3).}

\begin{tabular}{|l|l|l|}
\hline Data & Sub-Data & Examples \\
\hline $\begin{array}{l}\text { Transporta- } \\
\text { tion Data }\end{array}$ & $\begin{array}{l}\text { Address } \\
\text { Data }\end{array}$ & $\begin{array}{l}\text { Address Line, Postal Code, } \\
\text { City, Country }\end{array}$ \\
\cline { 2 - 3 } & $\begin{array}{l}\text { Supplier } \\
\text { Data }\end{array}$ & $\begin{array}{l}\text { Courier Name, Unique } \\
\text { Code of Courier/Courier } \\
\text { ID }\end{array}$ \\
\hline $\begin{array}{l}\text { Notifica- } \\
\text { tions Data }\end{array}$ & $\begin{array}{l}\text { Contact } \\
\text { Data }\end{array}$ & $\begin{array}{l}\text { Phone Numbers, E-Mail } \\
\text { Lists, E-Mail Addresses, } \\
\text { iOs Device ID, Google ID }\end{array}$ \\
\hline Event Data & $\begin{array}{l}\text { Check- } \\
\text { point Data }\end{array}$ & $\begin{array}{l}\text { Promised Delivery Date, } \\
\text { Date and Time of Order } \\
\text { Created, Date and Time of } \\
\text { Pick up, Date and Time of } \\
\text { Delivery }\end{array}$ \\
\hline
\end{tabular}

The transportation categories contain transportation data, time data, event data, and tracking numbers (including the tracking status and shipment information). Transportation data includes data on the modality. They are comprised of the transportation mode, i.e., street, sea, air, or rail, and the supplier data. They describe how the logistical task is fulfilled and by whom. The next category is time data. These data contain various information on

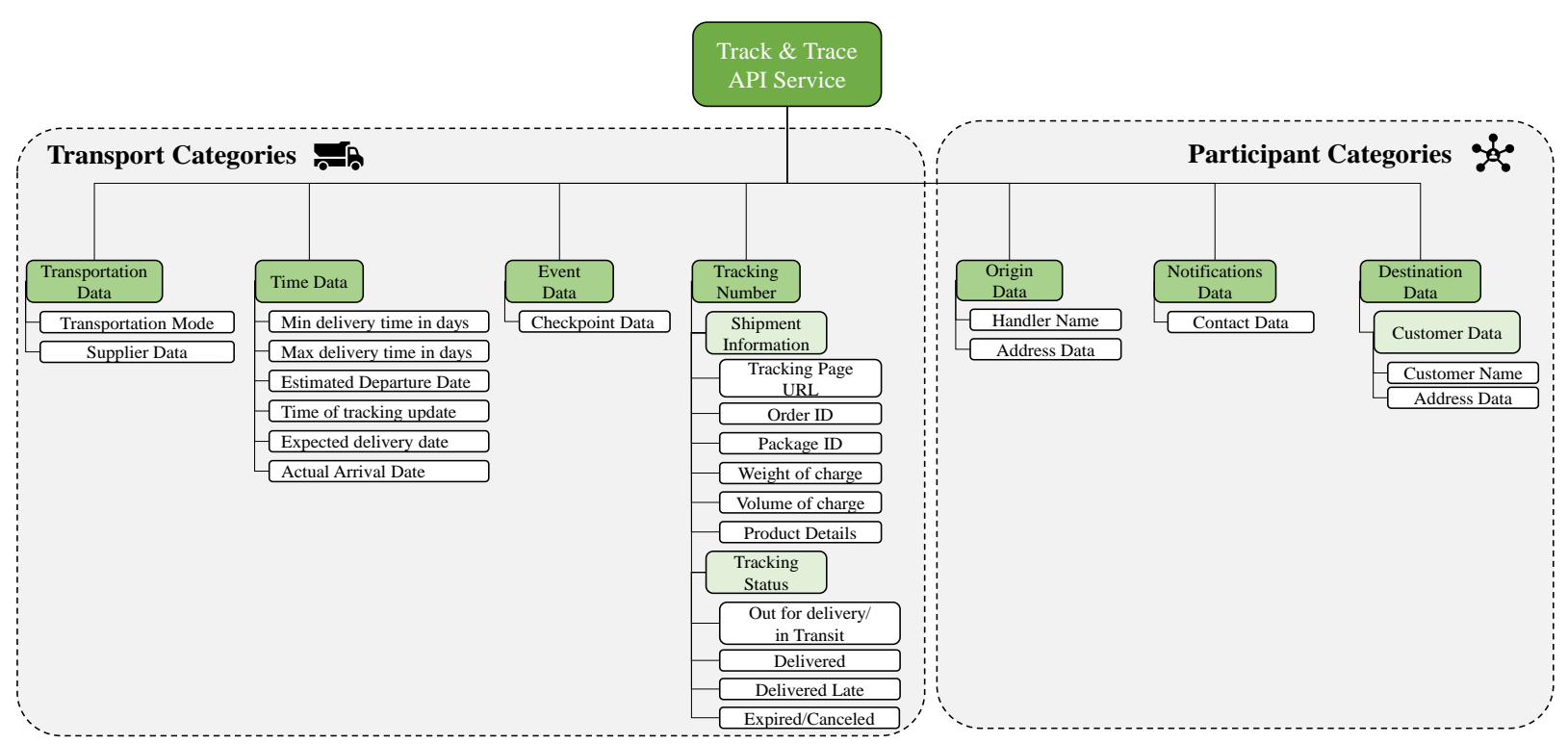

Figure 3. Taxonomy of data for track \& trace API services. 
when something happens during the transport, i.e., deviations from a priori agreed parameters. Data types are minimal delivery times and maximal delivery times, estimated departure dates, times of updates (see event/checkpoint data), expected delivery updates, and actual arrival dates. This information may be input and output data, as delivery times are equally crucial for the calculation and results of any track and trace process. Also, there are the event data. That compromises the checkpoint data (see Table 6), e.g., when transportation vehicles reach specific points on their route. Especially on overseas transports, there is no complete coverage of tracking possibilities, as sensors do not connect to servers while on seas. Here, reaching checkpoints are opportunities to send data.

Last, the category tracking number, which connects the shipment information and the tracking status. Part of the shipment information is the tracking page URLs, the order and package IDs, and the weight, volume, and further details of the transported goods. These data describe the transported good and provides, first of all, additional information. Crucial for the trace of the goods are the individual package $I D s$ and the tracking page URLs, as these offer the opportunity to gain access to the good's information. As a second sub-category, there is the tracking status, which describes the actual state of the delivery. It contains the states out of delivery / in transit, the state delivered, the possibility of a late delivery, and the remark expired/canceled. The status is often an output of any track and trace process and summarizes the important information. Together with the time data, track and trace enable transparency over the logistical process and allow further planning and optimization.

The first category in participants is origin data, which includes data about the point of origin the transport is shipped from. Precisely, it consists of the handler name and address data. Address data include all sub-data necessary to describe the point of origin. For example, these include postal codes, cities, or countries (see Table 6).

Next, these data form the necessary information for the contact and notifications data. Of high importance are telephone numbers to call in case of contact, electronic addresses and IDs, i.e., mail, iOs, or Google accounts, and overviews of a contact person.

\section{Discussion, Contributions, and Limitations}

The paper proposes three taxonomies that are engraved in a larger research project and reflect our current progress. The 'service-level' taxonomy (see Figure 1) organizes services API services for logistics.
Since API services can be classified as data-driven services, they naturally require data resources to work. We draw from the logic of establishing conceptual hierarchies between taxonomies, i.e., a more generic view (on API services) and a specific view (on data required for a related service) [20, 21]. Subsequently, the taxonomy for track \& trace (see Figure 3) illustrates én detail which data are retrievable and useable through APIs in logistics. Indeed, this is not a prescription that all such data should be used. Instead, it is an organization of potential data resources that logistics service providers offering track \& trace can use via APIs. Taxonomy users can discover new potentials based on these data for their track \& trace service. For example, logistics service providers could check whether offering extended services on not-yetleveraged data resources (e.g., time of tracking updates) to enhance their services. That logic also applies to the taxonomy for route optimization data (see Figure 2). It enables its users to find new parameters that can be used to achieve more efficient route optimization or offer comprehensive services. For example, route optimizers can integrate data about road infrastructure (e.g., rest areas) or constant road restrictions (e.g., seasonal closures or existing bridges or tunnels en route) to optimize the routing engine for better results.

In terms of research contributions, our work complements existing research on digital and datadriven business models and corresponding services in the logistics industry (see $[6,18]$ ). Because of that, it is an industry-specific, detailed look at APIs in logistics, enabling researchers to scope the field better and systemize it more profoundly. It also is a potential starting point to transfer these findings onto other domains and spur research on data-driven innovation. On a more general notion, our work addresses one particular channel of data transfer that, arguably, will become more critical during the course of the ongoing digitalization of industry and society.

For practitioners, our taxonomy contributes possibilities to leverage APIs either as a consumer or provider. While we cannot claim completeness, it still gives practitioners an overview of available APIs and their implications for services. They can identify opportunities to collect data, use them for new services or identify potential white spots that they can fill with their data. We see high potential for practitioners to extend, enrich, or develop altogether new business models based on data. The taxonomies give practitioners checklists for which data to consider. At this point, it does so for track \& trace services and route optimization services. Practically, that means that the users of the taxonomy can identify new potentials for data. 
Our work has limitations. First, we collected the data from three API databases based on publicly available data at a specific period in time. Meaning, our work can only look in from the 'outside' and give a snapshot in time of the currently available APIs. Next, since analyzing and abstracting the information requires a degree of judgment, others might identify other dimensions as more essential. Also, there might be more API databases available on the market that we did not include in our current sample. Because of that, our taxonomy is only an excerpt based on the dataset we collected in our work. Lastly, our taxonomy needs to be developed in future work to complement other services through additional lower-level taxonomies (e.g., as we propose here with route optimization and track \& trace).

Our work paves the way for further research. The taxonomies are only an excerpt of the landscape of APIs based on public data analysis. Future work should include additional methods of inquiry to extend our results. Firstly, incorporating other databases or searching explicitly for APIs of logistics companies can identify additional samples. For example, software databases often indicate whether a company offers an API (e.g., GetApp ${ }^{6}$ or Sourceforge $e^{7}$ ). Those databases potentially contain information on APIs directly or give hints on which software (e.g., through their websites) to analyze in more detail. Second, adapting the underlying methodological approach to shift from analyzing publicly available data to inquiring directly with logistics firms, e.g., through interviews or questionnaires. Adopting that approach would also be a potential avenue to further complement our findings through experiences and best practices from practitioners. Third, a more comprehensive look at what data 'are available' or 'needed' in the logistics industry could rationalize new API-based services. Lastly, a novel mode of inquiry could be the automated classification of APIs and their services through a machine learning approach.

\section{Acknowledgment}

This research was conducted in the project "Silicon Economy Logistics Ecosystem" and is funded by the German Federal Ministry of Transport and Digital Infrastructure.

\section{References}

[1] van Marwyk, K. and S. Treppte, 2016 Logistics Study on Digital Business Models, Roland Berger, 2016.

[2] Bauer, I. and A. Wortman, Transport and logistics barometer: 2020 mid-year analysis of M\&A deals, joint

\footnotetext{
${ }^{6} \mathrm{https}: / /$ www.getapp.de/ last-accessed: 28-08-2021
}

ventures and strategic alliances in the transport and logistics industry, PWC, 2020.

[3] Cichosz, M., C.M. Wallenburg, and A.M. Knemeyer, "Digital transformation at logistics service providers: barriers, success factors and leading practices", The International Journal of Logistics Management, 31(2), 2020, pp. 209-238.

[4] Hofmann, E. and F. Osterwalder, "Third-Party Logistics Providers in the Digital Age: Towards a New Competitive Arena?", Logistics, 1(2), 2017, pp. 1-28.

[5] Klötzer, C. and A. Pflaum, "Toward the Development of Maturity Model for Digitalization within the Manufacturing Industry's Supply Chain", Proceedings of the 50th Hawaii International Conference on System Sciences, 2017.

[6] Möller, F., H. Bauhaus, C. Hoffmann, C. Niess, and B. Otto, "Archetypes of Digital Business Models in Logistics Start-Ups", in Proceedings of the 27th European Conference on Information Systems. 2019: Uppsala, Stockholm.

[7] Göpfert, I. and P. Seeßle, Startup-Unternehmen in der Logistikbranche: Marktübersicht und aktuelle Entwicklung junger innovativer LogistikUnternehmen(06), 2018.

[8] Iyer, B. and M. Subramaniam, "Are You Using APIs to Gain Competitive Advantage?", Harvard Business Review, 13, 2015.

[9] Wulf, J. and I. Blohm, "Service Innovation through Application Programming Interfaces - Towards a Typology of Service Designs", in Proceedings of the 38th International Conference on Information Systems, Seoul: South Korea. 2017: Seoul: South Korea.

[10] Um, S.Y., Y. Yoo, S. Wattal, R.J. Kulathinal, and B. Zhang, "The Architecture of Generativity in a Digital Ecosystem: A Network Biology Perspective", International Conference on Information Systems (ICIS 2013): Reshaping Society Through Information Systems Design, 4, 2013, pp. 3721-3733.

[11] Evans, P.C. and R.C. Basole, "Revealing the API ecosystem and enterprise strategy via visual analytics", Communications of the ACM, 59(2), 2016, pp. 26-28.

[12] Tilson, D., K. Lyytinen, and C. Sørensen, "Research Commentary - Digital Infrastructures: The Missing IS Research Agenda", Information Systems Research, 21(4), 2010, pp. 748-759.

[13] Iyer, B. and M. Subramaniam, "The Strategic Value of APIs", Harvard Business Review, 2015.

[14] https://www.programmableweb.com/about, accessed 828-2021.

[15] Geissbauer, R., J. Vedso, and S. Schrauf, Industry 4.0: Building the digital enterprise, 2016.

[16] https://www.dhl.com/global-en/home/insights-andinnovation/insights/logistics-trend-radar.html, accessed 6-12-2021.

[17] Vukovic, M., B. Srivastava, J.W. Branch, J. Laredo, V. Muthusamy, A. Slominski, R. Vaculin, W. Tan, V. Naik, I. Silva-Lepe, and A. Kumar, "Riding and thriving on the API hype cycle", Communications of the ACM, 59(3), 2016, pp. 35-37.

\footnotetext{
${ }^{7}$ https://sourceforge.net/ last-accessed: 28-08-2021
} 
[18] Möller, F., M. Stachon, C. Hoffmann, H. Bauhaus, and B. Otto, "Data-driven Business Models in Logistics: A Taxonomy of Optimization and Visibility Services", in Proceedings of the 53rd Hawaii International Conference on System Sciences. 2020: Hawaii: USA.

[19] Möller, F., T. Guggenberger, and B. Otto, "Design Principles for Route-Optimization Business Models: A Grounded Theory Study of User Feedback", in Proceedings of the 15th International Conference on Wirtschaftsinformatik, Potsdam: Germany. 2020.

[20] Glass, R.L. and I. Vessey, "Contemporary ApplicationDomain Taxonomies", IEEE Software, 12(4), 1995, pp. 63-76.

[21] Hanelt, A., B. Hildebrandt, and J. Polier, "Uncovering the Role of IS in Business Model Innovation - A Taxonomy-Driven Approach to Structure the Field", in Proceedings of the 23rd European Conference on Information Systems, Münster: Germany. 2015.

[22] Kamprath, M. and B. Halecker, "A Systematic Approach for Business Model Taxonomy-How to operationalize and compare large Quantities of Business Models?", in ISPIM Innovation Symposium, Seoul: South Korea. 2012.

[23] Yoo, Y., O. Henfridsson, and K. Lyytinen, "Research Commentary - The New Organizing Logic of Digital Innovation: An Agenda for Information Systems Research", Information Systems Research, 21(4), 2010, pp. 724-735.

[24] Lambert, S., "The Importance of Classification to Business Model Research", Journal of Business Models, 3(1), 2015, pp. 49-61.

[25] Baden-Fuller, C. and M.S. Morgan, "Business Models as Models", Long Range Planning, 43(2-3), 2010, pp. 156-171.

[26] Nickerson, R.C., U. Varshney, and J. Muntermann, "A Method for Taxonomy Development and its Application in Information Systems", European Journal of Information Systems, 22(3), 2013, pp. 336-359.

[27] Osterwalder, A., Y. Pigneur, and C.L. Tucci, "Clarifying Business Models: Origins, Present, and Future of the Concept", Communications of the Association for Information Systems, 16(1), 2005, pp. 1-25.

[28] Schüritz, R., S. Seebacher, and R. Dorner, "Capturing Value from Data: Revenue Models for Data-Driven Services", in Proceedings of the 50th Hawaii International Conference on System Sciences, Hawaii: USA. 2017.

[29] Guggenberger, T.M., F. Möller, K. Boualouch, and B. Otto, "Towards a Unifying Understanding of Digital Business Models", in Proceedings of the Twenty-Third Pacific Asia Conference on Information Systems, Dubai: UAE. 2020.

[30] Hartmann, P.M., M. Zaki, N. Feldmann, and A. Neely, "Capturing Value from Big Data - A Taxonomy of Data-Driven Business Models Used by Start-Up Firms", International Journal of Operations \& Production Management, 36(10), 2016, pp. 13821406.

[31] Azkan, C., L. Iggena, I. Gür, F. Möller, and B. Otto, "A Taxonomy for Data-Driven Services in Manufacturing
Industries", in Proceedings of the 24th Pacific Asia Conference on Information Systems, Dubai: UAE. 2020.

[32] Heinbach, C., S. Hagen, and O. Thomas, "FreightLogistics-as-a-Service - Innovative Geschäftsmodelle für ein datengetriebenes Transportmanagement", HMD Praxis der Wirtschaftsinformatik, 58(3), 2021, pp. 580594.

[33] Gruchmann, T., N. Pratt, J. Eiten, and A. Melkonyan, "4PL Digital Business Models in Sea Freight Logistics: The Case of FreightHub", Logistics, 4, 2020, pp. 1-15.

[34] Mikl, J., D.M. Herold, M. Ćwiklicki, and S. Kummer, "The impact of digital logistics start-ups on incumbent firms : a business model perspective", The International Journal of Logistics Management, 2020.

[35] Souza, V., R. Cruz, W. Silva, S. Lins, and V. Lucena, "A Digital Twin Architecture Based on the Industrial Internet of Things Technologies", in 2019 IEEE International Conference on Consumer Electronics (ICCE), 2019 IEEE International Conference on Consumer Electronics (ICCE), Las Vegas, NV, USA, 11.01.2019 - 13.01.2019. IEEE.

[36] Ghazawneh, A. and O. Henfridsson, "Balancing platform control and external contribution in thirdparty development: the boundary resources model", Information Systems Journal, 23(2), 2013, pp. 173192.

[37] Lin, A. and N.-C. Chen, "Cloud computing as an innovation: Percepetion, attitude, and adoption", International Journal of Information Management, 32(6), 2012, pp. 533-540.

[38] Pautasso, C., O. Zimmermann, and F. Leymann, "Restful web services vs. "big"' web services", in Proceedings of the 17th International Conference on World Wide Web. 2008: Beijing, China.

[39] Jacobson, D., G. Brail, and D. Woods, APIs: A Strategy Guide, O'Reilly Media, 2011.

[40] Wulf, J. and I. Blohm, "Service Innovation through Application Programming Interfaces - Towards a Typology of Service Designs", Thirty Eighth International Conference on Information Systems,, 2017.

[41] Wulf, J. and I. Blohm, "Fostering Value Creation with Digital Platforms: A Unified Theory of the Application Programming Interface Design", Journal of Management Information Systems, 37(1), 2020, pp. 251-281.

[42] https://rapidapi.com/, accessed 6-16-2021.

[43] https://datarade.ai/, accessed 6-16-2021.

[44] Szopinski, D., T. Schoormann, and D. Kundisch, "Visualize Different: Towards Researching the Fit Between Taxonomy Visualizations and Taxonomy Tasks", in Proceedings of the 15th International Conference on Wirtschaftsinformatik. 2020: Potsdam: Germany. 